\title{
Use of Microscopic Theoretical Methods for The Construction of The Nuclear Wavefunction of ${ }^{7} \mathrm{Li}$
}

\author{
Neelam Sinha ${ }^{1} \cdot$ Piyush Sinha ${ }^{2 *}$ \\ ${ }^{1}$ Dept. of Physics, S.D. College, Muzaffarnagar \\ ${ }^{2}$ Dept. of Physics, H.N.B. Garhwal University, B.G.R Campus, Pauri \\ *Corresponding Author Email: piyushs03@gmail.com \\ Received: 4.9.2021; Revised: 19.10.2021; Accepted: 28.10.2021 \\ CSociety for Himalayan Action Research and Development
}

\begin{abstract}
Microscopic theoretical studies of scattering and reaction problems for light nuclei have been extensively carried out using resonating group method. In this paper we have used the nuclear cluster model, the resonating group method, the generator coordinate method and complex generator coordinate technique for the construction of microscopic antisymmetrized nuclear wavefunction of ${ }^{7} \mathrm{Li}$ nucleus. This wavefunction can be further used to calculate the structural properties of the nucleus. The ${ }^{7} \mathrm{Li}$ nucleus in ground state is considered as a nuclear system consisting of three clusters namely an alpha cluster, a deuteron cluster and a neutron cluster. We have chosen spatial, spin and isospin function of cluster internal functions. The arguments of internal wavefunction include the parameter coordinates. These parameters can be adjusted to some extent to obtain predictions close to experimental results. The wavefunction is written using shell model with definite parity and angular momentum. The complex generator coordinate technique allows this wavefunction to write it as an antisymmetrized product of seven single particle functions after inclusion of the wavefunction for the center- of -mass motion.
\end{abstract}

Key words: Cluster Model • Resonating Group Method • Complex Generator Coordinate Technique

\section{Introduction}

${ }^{7} \mathrm{Li}$ is a stable nucleus in ground state and its natural abundance is 92.41 percent. In cluster model (Wildermuth and et al, 1958) nucleons are separated into several groups and constitute clusters and exchangefrom one cluster to another. According to resonating group method (Wildermuth, 1977), we have considered internal wavefunction of each nucleon and the complete antisymmetrization is performed to describe indistinguishability of nucleons between different clusters. ${ }^{7} \mathrm{Li}$ nucleus consists of 3 protons and 4 neutrons in ground state and is assumed to be made up of three clusters (Margenau, 1941 and Brink
1968), an alpha cluster (two protons and two neutrons), a deuteron cluster (one proton and one neutron) and a neutron (single neutron) cluster. The spin and parity of the nucleus in ground state is $\mathrm{J}^{\pi}=\frac{3^{-}}{2}$. In the nuclear shell model (F.C. Khanna, 1968 and Stovall, 1969), for the structure of the nucleus a mean field potential inside the atomic nucleus is used to describe approximately the forces applied by nucleons on each other is named Wood-Saxon potential (T.W. Donnelly et.al, 1969).

\section{Wavefunction of ${ }^{7} \mathrm{Li}$ ground state using complex generator coordinate technique}

The cluster model wavefunction (K. Wildermuth.et.al, 1966) of $7 \mathrm{Li}$ nucleus 
including the center-of-mass wavefunction $\mathrm{Z}\left(\overline{\mathrm{R}}_{\mathrm{cm}}\right)$ can be written as:

$$
\Phi_{\frac{\mathrm{g}}{\mathrm{M}}}=\mathrm{A}\left[\Phi(\alpha) \Phi(\mathrm{d}) \chi_{\chi_{\ln }}\left(\overline{\mathrm{R}}_{\alpha}^{\prime}-\overline{\mathrm{r}}_{\mathrm{d}}^{\prime}\right)_{\chi_{\ln \mathrm{l}}}\left(\overline{\mathrm{R}}_{\alpha}^{\prime}-\overline{\mathrm{R}}_{7}^{\prime}\right)\right.
$$

Here $\mathrm{A}$ is the antisymmetrization operator, and $\Phi(\alpha), \Phi(d)$ are wavefunctions of alpha and deuteron cluster, $\chi_{\ln \rrbracket}$ relative motion wavefunction and $\mathrm{Z} \quad\left(\overline{\mathrm{R}}_{\mathrm{cm}}\right)$ center-of-mass wavefunction.
To convert this wavefunction into antisymmetrized product of single-particle wavefunction introducing an integral representation for $\Phi$, one gets

$$
\begin{aligned}
& \Phi_{\mathrm{g}_{\mathrm{M}}}=\mathrm{A} \int \Phi_{\mathrm{s}}\left(\alpha, \mathrm{R}_{\alpha}^{\prime}\right) \delta\left(\overline{\mathrm{R}}_{\alpha}-\overline{\mathrm{R}}_{\mathrm{d}}^{\prime}\right) \Phi_{\mathrm{s}}\left(\mathrm{d}, \mathrm{R}_{\mathrm{d}}^{\prime}\right) \delta\left(\overline{\mathrm{R}}_{\mathrm{d}}-\overline{\mathrm{R}}_{\mathrm{d}}^{\prime}\right) \delta\left(\overline{\mathrm{r}}_{7}-\overline{\mathrm{r}}_{7}^{\prime}\right) \\
& \xi_{\alpha} \xi_{\mathrm{d}} \xi_{\mathrm{n}} \chi_{\mathrm{lm} \mathrm{l}}\left(\overline{\mathrm{R}}_{\alpha}^{\prime}-\overline{\mathrm{R}}_{\mathrm{d}}^{\prime}\right) \cdot \chi_{\mathrm{lm} \mathrm{l}}\left(\overline{\mathrm{R}}_{\alpha}^{\prime}-\overline{\mathrm{r}}_{7}^{\prime}\right) \mathrm{Z}_{\mathrm{cm}}\left(\frac{\left.4 \overline{\mathrm{R}}_{\alpha}^{\prime}+2 \overline{\mathrm{R}}_{\mathrm{d}}^{\prime}+\overline{\mathrm{r}}_{7}^{\prime}\right) \mathrm{dR}_{\alpha}^{\prime} \mathrm{dR}_{\mathrm{d}}^{\prime} \mathrm{dr} \mathrm{r}_{7}^{\prime}}{}\right.
\end{aligned}
$$

where we have chosen, $\Phi$ for alpha and deuteron cluster as the product of spatial part and spin isospin part i.e.,

$$
\Phi=\Phi_{s} \xi(\sigma, \tau)
$$

$\chi_{\ln \sqrt{1}}\left(\mathrm{R}_{\alpha}^{\prime}-\mathrm{R}_{\mathrm{a}}^{\prime}\right)$ is the relative motion wavefunction between alpha and deuteron cluster, $\chi_{\ln \mathrm{I}}\left(\mathrm{R}_{\alpha}^{\prime}-\mathrm{R}_{\tau}^{\prime}\right)$ is the relative motion wavefunction between alpha and neutron.

Here the choice for ' 1 ' is takenas $J=\frac{3}{2}$ and the of spin of seventh nucleon is $\frac{1}{2}$ so ' 1 ' can have possible value 1 or 2 but as parity of the nucleus is negative, so ' 1 ' must be odd i.e., ' 1 ' $=1$ which gives $\mathrm{m}_{1}=1,0,-1$.

For $J=\frac{3}{2}$ the magnetic quantum number may have four values $\frac{3}{2}, \frac{1}{2},-\frac{1}{2},-\frac{3}{2}$. Corresponding to these values one can write relative motion wavefunction between alpha and deuteron cluster is

$$
\chi_{11}(R)=|R| Y_{11}(\widetilde{R}) \exp \left(\frac{-4}{9} \beta R^{2}\right)
$$

and the relative motion wavefunction between alpha and neutron cluster is:

$$
\chi_{10}(R)=|R| Y_{10}(R) \exp \left(\frac{-4}{9} \beta R^{2}\right)
$$


where

$$
\begin{aligned}
& \mathrm{Y}_{11}(\mathrm{R})=-\sqrt{\frac{3}{8 \pi}} \frac{(\mathrm{x}+\mathrm{iy})}{\llbracket \mathrm{R}\rceil} \\
& \mathrm{Y}_{10}(\mathrm{R})=-\sqrt{\frac{3}{4 \pi}} \frac{\mathrm{z}}{|\mathrm{R}|}
\end{aligned}
$$

in eq. (1.2) introducing following representation for the delta function

$$
\delta\left(\mathrm{R}_{\mathrm{K}}-\mathrm{R}_{\mathrm{K}}^{\mathrm{m}}\right)=\left(\frac{1}{2 \pi}\right)^{3} \int \exp \left[\mathrm{i} \mathrm{S}_{\mathrm{K}}^{\mathrm{m}} \cdot\left(\overline{\mathrm{R}}_{\mathrm{K}}-\overline{\mathrm{R}}_{\mathrm{K}}^{\mathrm{m}}\right)\right] \mathrm{dS}_{\mathrm{K}}^{\mathrm{m}}
$$

where $\mathrm{K}=$ alpha cluster and deuteron cluster

$\mathrm{S}_{\mathrm{K}}^{\mathrm{I}}$ denotes generator coordinate. We can write $\delta$ function for seventh nucleon as:

$\delta\left(\mathrm{r}_{7}-\mathrm{r}_{7}^{\mathrm{m}}\right)=\left(\frac{\alpha}{4 \pi}\right)^{3} \int \exp \left[\frac{-\alpha}{2}\left(\overline{\mathrm{r}}_{7}-\frac{\mathrm{i}}{2} \overline{\mathrm{S}}_{7}^{\mathrm{m}}\right)^{2}+\frac{\alpha}{2}\left(\overline{\mathrm{r}}_{7}^{\mathrm{m}}-\frac{\mathrm{i}}{2} \overline{\mathrm{S}}_{7}^{\mathrm{m}}\right)^{2}\right] \mathrm{d} \overline{\mathrm{S}}_{7}^{\mathrm{m}}---(1.9)$

substituting eqs. from (1.3) to eqn. (1.9) in eqn. (1.2) and using Jacobian of transformation $|\mathrm{J}|$ $\mathrm{d}_{\mathrm{R}}^{\mathrm{m}} \mathrm{d} \overline{\mathrm{R}}_{\mathrm{d}}^{\mathrm{m}} \mathrm{d} \overline{\mathrm{r}}_{7}^{\mathrm{m}} \mathrm{d} \overline{\mathrm{S}}_{\alpha \mathrm{m}}^{\mathrm{m}} \mathrm{d} \overline{\mathrm{S}}_{\mathrm{d}}^{\mathrm{m}} \mathrm{d} \overline{\mathrm{S}}_{7}^{\mathrm{m}}=|\mathrm{J}| \mathrm{d} \overline{\mathrm{R}}_{1}^{\mathrm{m}} \mathrm{d} \overline{\mathrm{R}}_{2}^{\mathrm{m}} \mathrm{dR} \mathrm{R}_{\mathrm{cm}}^{\mathrm{m}} \mathrm{d} \overline{\mathrm{P}} \mathrm{d} \overline{\mathrm{Q}} \mathrm{d} \overline{\mathrm{S}}_{7}^{\mathrm{m}}$

Also we have chosen center-of-mass wavefunction as

$$
\mathrm{Z}\left(\overline{\mathrm{R}}_{\mathrm{cm}}^{\mathrm{m}}\right)=\exp \left(\frac{-7}{2} \alpha \mathrm{R}_{\mathrm{cm}}^{\mathrm{m} 2}\right)
$$

The eq. (1.2) will have the final form

$$
\begin{aligned}
& \Phi_{g \mathrm{~g}}=\mathrm{A} \int \prod_{\mathrm{J}=1 \mathrm{~J}}^{4} \prod_{\mathbb{K}=5}^{6} \xi_{\alpha} \xi_{\mathrm{d}} \xi_{\mathrm{n}} \exp \left\{\frac{-\alpha}{2}\left(\overline{\mathrm{r}}_{\mathrm{j}}-\overline{\mathrm{p}}\right)^{2}\right\} \\
& \exp \cdot\left\{\frac{-\alpha}{2}\left(\mathrm{r}_{\mathrm{K}}-\mathrm{iQ}\right)^{2}\right\} \exp \cdot\left\{\frac{-\alpha}{2}\left(\overline{\mathrm{r}}_{7}+4 \mathrm{i} \overline{\mathrm{p}}+2 \mathrm{iQ}\right)^{2}\right\}
\end{aligned}
$$

$$
\begin{gathered}
\chi_{11}\left(\overline{\mathrm{R}}_{1}^{\mathrm{m}}\right) \chi_{10}\left(\overline{\mathrm{R}}_{2}^{\mathrm{m}}\right) \exp \cdot\left[-2 \alpha\left\{(\overline{\mathrm{P}}+\overline{\mathrm{Q}})^{2}-2 \overline{\mathrm{P}} \cdot \overline{\mathrm{Q}}\right\}+\right. \\
\alpha \mathrm{R}_{1}^{\mathrm{m} 2}+\frac{4}{7} \alpha \mathrm{R}_{2}^{\mathrm{\prime \prime} 2}-2 \mathrm{i} \alpha \overline{\mathrm{R}}_{1}^{\mathrm{m}} \cdot(\overline{\mathrm{P}}-\overline{\mathrm{Q}})-\frac{36 \mathrm{i}}{7} \alpha \mathrm{R}_{2}^{\mathrm{m}} \cdot(\overline{\mathrm{P}}+\overline{\mathrm{Q}})-
\end{gathered}
$$




$$
8 \alpha(\overline{\mathrm{P}}+\overline{\mathrm{Q}})^{2} \mathrm{~d} \overline{\mathrm{P}} \mathrm{d} \overline{\mathrm{Q}} d R_{1}^{\mathrm{m}} \mathrm{dR}
$$

Where spin-isospin of ${ }^{7} \mathrm{Li}$ can be represented symbolically as:

$$
\begin{aligned}
\xi_{7 \mathrm{Li}}= & \xi_{\mathrm{\alpha}} \xi_{\mathrm{d}} \xi_{\mathrm{n}} \\
& =(\alpha v \alpha \pi \beta v \beta \pi: \alpha v \beta \pi: \alpha v) \\
\text { or } \quad & =\langle\uparrow \downarrow \uparrow \uparrow \uparrow \downarrow \downarrow: \uparrow \downarrow \downarrow \uparrow: \uparrow \downarrow\rangle
\end{aligned}
$$

The wavefunction represented by eq. (1.12) can be normalized by writing

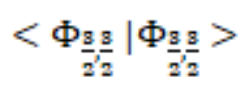

\section{Result and Discussion}

In this paper the emphasis is to develop a wavefunction for ${ }^{7} \mathrm{Li}$ nucleus using cluster model wavefunction along with the complex generator coordinate technique. In this method we have used parameter generator coordinates and width parameters $\alpha$ and $\beta$ for the alpha and deuteron clusters which can be adjusted by the calculations of binding energy. As the antisymmetrization (A. Herzenberg, 1957) of the wavefunction and motion of center-of-mass have been properly taken into account, the calculations of different parameters related to structure of nucleus like rms radius, quadrupole moment and the charge form factor may give good results. We have earlier calculated charge form factor of ${ }^{5} \mathrm{He}$ nucleus (P. Sinha, 2019 and P. Sinha et al, 2013) by using the same method and the calculations have yielded good results when compared with experimental data. The same approach has been employed successfully to construct the ground state wavefunction of ${ }^{6} \mathrm{Li}$ nucleus and ${ }^{9} \mathrm{Be}$ nucleus (P. Sinha, 2011 and N. Sinha, 2020). Studies of ${ }^{7} \mathrm{Li}$ are very useful as it is used as a hydroxide in controlling the chemistry of pressurized water reactor (PWR) cooling system and it is a key component of fluoride coolant in molten salt reactors where the coolant temperatures required are above of $800^{\circ} \mathrm{C}$.

\section{References}

Brink D.M. and Weignny A. (1968) Microscopic description of the Nuclear Cluster Theory Nucl. Phy. A 1201235.

Donnelly T.W. and Walker G.E., (1969) Predictions of Diffraction Minima in Elastic Electron Scattering Using Finite Nuclear Potentials Phys. Rev. Letters 221121. 
Herzenberg A., (1957) Studies in the $\alpha$-particle model of nuclei, Nucl. Phys.3 1.

Khanna, F.C. (1968) Nuclear Structure of $\mathrm{CA}^{40}$ and Elastic Scattering of 750-MeV electrons, Phys. Rev. Letters 20871.

Margenau, (1941) Interaction of alpha-particles, Phys. Rev.59 37.

Sinha P. (2011) Ground State Wave function of ${ }^{9} \mathrm{Be}$ using Resonating group method along with complex generator, Coordinate Technique, Int. J. Theor. Appl. Sci. 3(1): 76-77.

Sinha P. (2013), A normalized Cluster Model Wave function of ${ }^{5}$ Henucleus using CGCT. Int. J. of Theor. Appl. Sci. 5(2): 114-120.

Sinha P. and Sinha N. (2020) Cluster Shell Model Wave Function: Structure of ${ }^{6}$ Li Nucleus. J. Mountain Res. Vol. 15, 131-133

Stovall and Vinci Guerra D. (1969) Shell Model analysis of elastic e ${ }^{4}$ He Scattering Lettre at Nuo Vo Cim 1100.

Wildermuth K and Kanellopoulos (1958) Microscopic description of the Nuclear Cluster Theory Nucl. Phys. 7150.

Wildermuth K and Tang Y.C., (1977) A Unified Theory of the Nucleus, Physics Today 30762.

Wildermuth K. and Clure W., (1966) Cluster representation of nuclei, Springer Tracts in Modern Physics Vol. 41. 